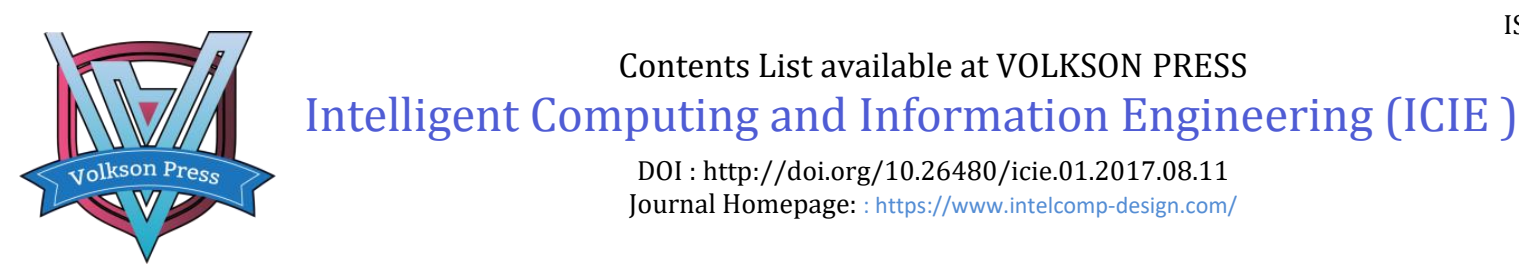

\title{
Simulation Research of Capacitance Sensor Based on Comsol
}

Li Cai ${ }^{1,{ }^{*}}$, Jianping Jia ${ }^{1}$ and Hongxia $\mathrm{Wu}^{1}$

${ }_{1}^{1}$ Mechanical \& Electrical Engineering College, Wuhan Donghu University, Wuhan *wherecl@163

This is an open access article distributed under the Creative Commons Attribution License, which permits unrestricted use, distribution, and reproduction in any medium, provided the original work is properly cited

\section{ARTICLE DETAILS}

\section{Article History:}

Received 12 May2017

Accepted 12 July 2017

Available online 14 September 2017

\section{Keywords:}

Capacitance sensor. Simulation research. Comsol software. Physical properties.

\section{ABSTRACT}

Comsol is widely used in various fields of scientific research and engineering calculation with convenient, and it can simulate all kinds of physical processes in the field of science and engineering, and realize the highly accurate numerical simulation. This paper, taking a capacitance sensor with two flat plates as object, first derives the theoretical basis of capacitance sensor, and describes physical properties of capacitance sensor. On this basis, this paper simulates the features of capacitance sensor considering the edge field and boundary condition by Comsol software. And simulation results show that electric field distribution under different air domain is obtained and the effectiveness of the simulation of capacitance sensor is verified. The simulation results provide a certain technical reference for the design of the subsequent capacitance sensor or the related detection system, which can prove that this software is an effective tool in the process of electromagnetic engineering field at the same time.

Effectiveness.

\section{Introduction}

Sensor is one of the most important parts in the measurement and control system. Therefore, it should be seen whether the characteristics parameters of the sensors meet the test requirements [1,2]. If there is no deviation or not, it must be adjusted or improved. It is necessary to analyze the characteristics of the sensor to determine whether the sensor meets the test requirements by the reflecting ability to the input quantity through the output value of the sensor. It is system identification process: first, the input and output data are observed, then the mathematical model of the studied object is extracted $[3,4]$. Real system identification methods are used in practice, it often need to carry out a lot of mathematical calculations. Usually, these operations cannot be achieved by hand quickly, which need to use the appropriate procedures, such as Comsol software, Matlab software and so on [5,6]. Comsol is a finite element numerical simulation software, which is widely used in various fields of scientific research and engineering calculation with convenient, easy to use, efficient, professional and other characteristics $[7,8]$. With high efficiency and good capability of multi field direct coupling analysis, it can simulate all kinds of physical processes in the field of science and engineering, and realize the highly accurate numerical simulation. It can find that the use of this software can bring great convenience to engineering testing, such as mathematical calculation or process simulation [9,10].

The typical capacitor consists of two conductors, and there is a medium between them. By applying the voltage difference between these objects, the electric field is formed. This is not only a direct electric field, but extends a distance, this phenomenon is called the edge of the field. In order to accurately predict the capacitance of the sensor, the edge field used in the domain model must be large enough and the appropriate boundary conditions must be used.

In view of improving the limitations, this paper, taking a capacitance sensor with two flat plates as object, proposes a capacitive sensor model with different air domains based on Comsol software. This model is influenced by the parallel plate capacitor and the size of air domain. Simulation results are discussed under different air domains, whose results show that it can be more convenient to directly familiar with the characteristics of components and to provide a reference for the follow-up testing system design. The chapters are as follows: Section 2 explains theoretical basis of capacitance sensor. Section 3 introduces simulation process, and then gives the simulation results. Section 4 summarizes the full text.

\section{THEORETICAL BASIS OF CAPACITANCE SENSOR}

Capacitive sensor is a kind of element with variable parameters. In most cases, the capacitance is composed of two parallel plates, whose medium is air. The capacitance of a capacitor consisting of two parallel plates is as follows:

$$
C=\frac{\varepsilon A}{d}
$$

where $\mathcal{E}$ is the dielectric constant of dielectric permittivity, $A$ is the covered area of the two plates, $d$ is the distance between two parallel plates, and $C$ is capacitance.

If we just change one parameter keeping other two parameters unchanged, the change of the parameter can be converted into the change of capacitance. Therefore, the variation of capacitance is proportional to the measured parameters. In practical use, parameter measurement is usually carried out by 
changing the distance between parallel plates, whose measurement sensitivity is higher than the sensitivity of the capacitive sensor by changing the other parameters.

The capacitance sensor can also be made into the detection device, according to the principle that the material dielectric constant is different, the measured capacitance value is different. The medium in the electric field will cause the electric field to change, which is due to the polarization of the molecule under the action of the electric field. An electric dipole moment is appeared in the medium, the electric field of the dipole moment is superimposed on the original electric field, which makes the electric field change. The electric flux density in the electrostatic field can be expressed as:

$$
D=\varepsilon E=\varepsilon_{0} \varepsilon_{r} E=\varepsilon_{0}\left(1+\frac{P}{\varepsilon_{0} E}\right) E=\varepsilon_{0} E+P
$$

where $\varepsilon_{r}$ is relative dielectric constant, which indicates the extent of change: the intensity of the dielectric changes the flux density; ${ }^{\varepsilon_{0}}$ is the dielectric constant of free space, which is polarization strength of medium; $D$ is the electric flux density.

\section{SIMULATION ANALYSIS}

The basic steps of the Comsol operation are as follows: First, the appropriate simulation module is selected, and the known parameters and the necessary boundary conditions are listed. Second, a geometric model is established according to the size. Third, the appropriate size of the grid is selected to divide the grid. Fourth, the material property parameters and the calculation constant are set. Fifth, calculation solving order is executed after setting the boundary conditions and physical parameters. Here, we select 'Electrostatics' in the select physics tree and then select 'Stationary' in study type tree. In the cylinder settings window, the radius is $10 \mathrm{~cm}$ and the height is $0.5 \mathrm{~cm}$. Radius of air domain is set respectively to $15 \mathrm{~cm}, 25 \mathrm{~cm}, 35 \mathrm{~cm}$ in three cases.

Figure 1 shows the capacitor consisting of two metal plates, which is in an air domain. The size of the sphere truncates the modeling space. This model studies the effect upon the capacitance with the size change of this air domain. One of the plates is designated as the ground with a voltage of $0 \mathrm{~V}$. The other plate has a voltage difference between the two plates with a voltage of $1 \mathrm{~V}$. The difference affects the capacitance and electric field intensity; the voltage itself is arbitrary.

The air sphere boundary can be considered to be one of two different physical situations: It can be seen as a fully insulating surface, which cannot be in charge redistribution itself, or completely conductive surface, over which the potential will not be different. The modeling realization of the perfectly insulating surface is the Zero Charge boundary condition, which also means the boundary tangential electric field line. The realization of the model fully conductive surface boundary conditions of the Floating Potential boundary condition. This voltage of all of the boundaries of the sphere is an unknown constant, which is computed during the solution. The boundary condition means that the electric field lines are perpendicular to the boundary. When studying the result of the convergence on the surrounding domain size, it is important to fix the element size. In this model, the size of the element is fixed as the domain size is different.

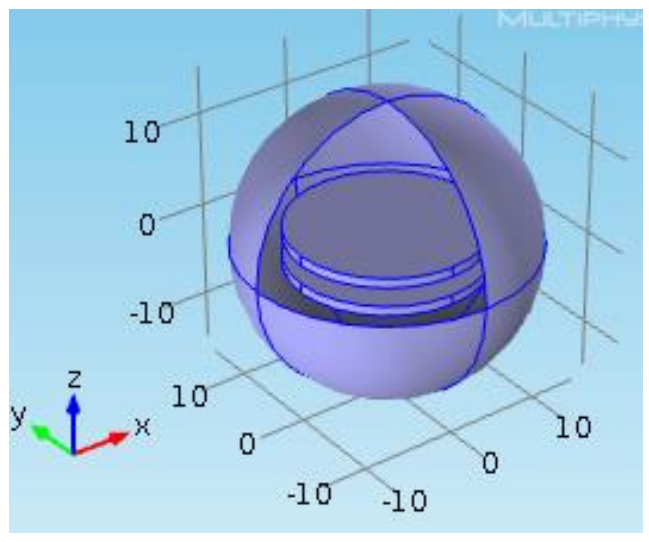

a

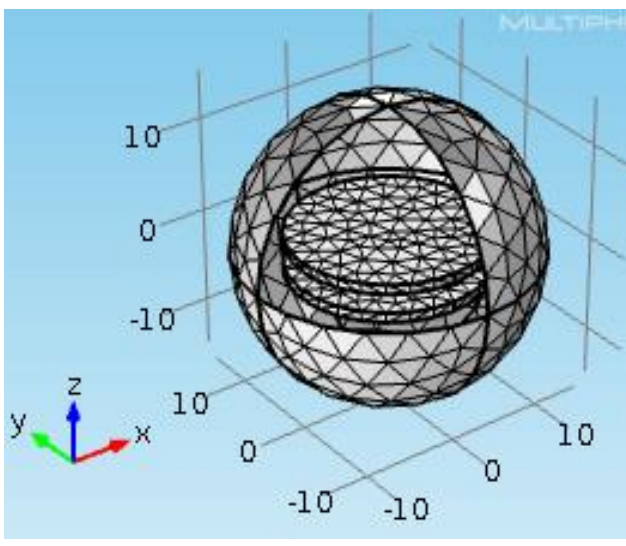

$\mathrm{b}$

Figure 1: Schematic diagram of the PSO algorithm

$$
\text { a- geometric model b- meshing }
$$

Figure 2 and Figure 3 plot the electric field cases where the air sphere boundary is treated as completely perfect. The fields terminate on the boundaries of the air sphere. In fig. 2, the electric field norm is represented by multiplies and electric field is represented by arrows under the Zero Charge boundary condition, compares the variation under different radius of the air domain, such as the air domain radius of $15 \mathrm{~cm}, 25 \mathrm{~cm}, 35 \mathrm{~cm}$. In figure 3 , the electric field norm and electric field under different coordinate perspective when the radius of air domain is $35 \mathrm{~cm}$.

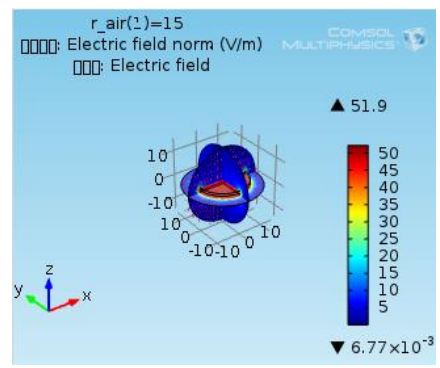

$\mathrm{a}$

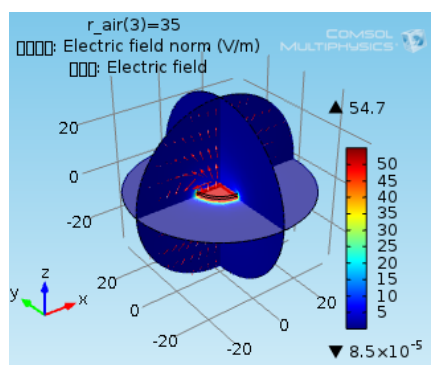

$\mathrm{b}$

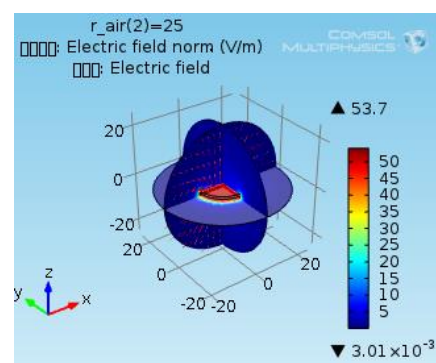

C 
Figure 2: The electric field norm (multislices) and electric field (arrows) for the case of the Zero Charge boundary condition with different radius of air domain

$$
\text { a- } \mathrm{r}=15 \mathrm{~cm} ; \quad \text { b- } \mathrm{r}=25 \mathrm{~cm} ; \quad \text { c- } \mathrm{r}=35 \mathrm{~cm}
$$

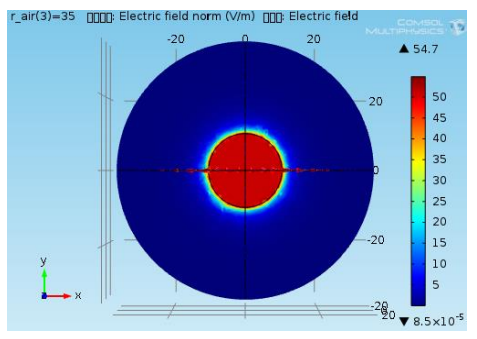

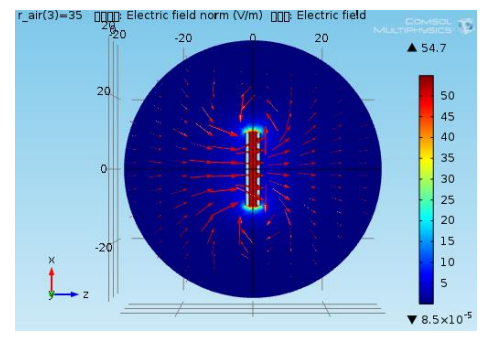

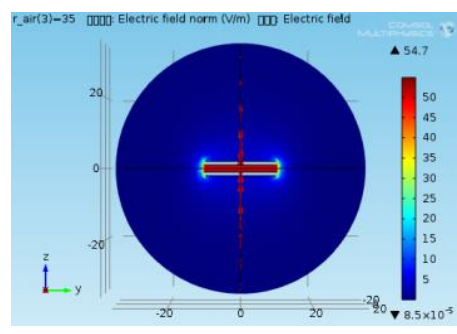

C

Figure 3: The electric field norm and electric field with the air domain radius of $35 \mathrm{~cm}$ under different coordinate perspective $\mathrm{a}$ - In the $\mathrm{x}$, y coordinates; $\mathrm{b}$ - In the $\mathrm{x}, \mathrm{z}$ coordinates; $\mathrm{c}$ - In the $\mathrm{y}$, $\mathrm{z}$ coordinates

Figure 4 compares the capacitance values of the device with respect to air sphere radius for the two boundary conditions. In fig. 4, electric insulation and fixed voltage boundary conditions converge to the same result. Among them, the green curve represents zero voltage condition, and the blue curve represents fixed voltage boundary condition. If a small air sphere model is built with the electric insulation and floating potential boundary conditions, we can obtain the average of the two conditions, which also represented by red curve. All three capacitance calculations converge to the same value as the radius grows.

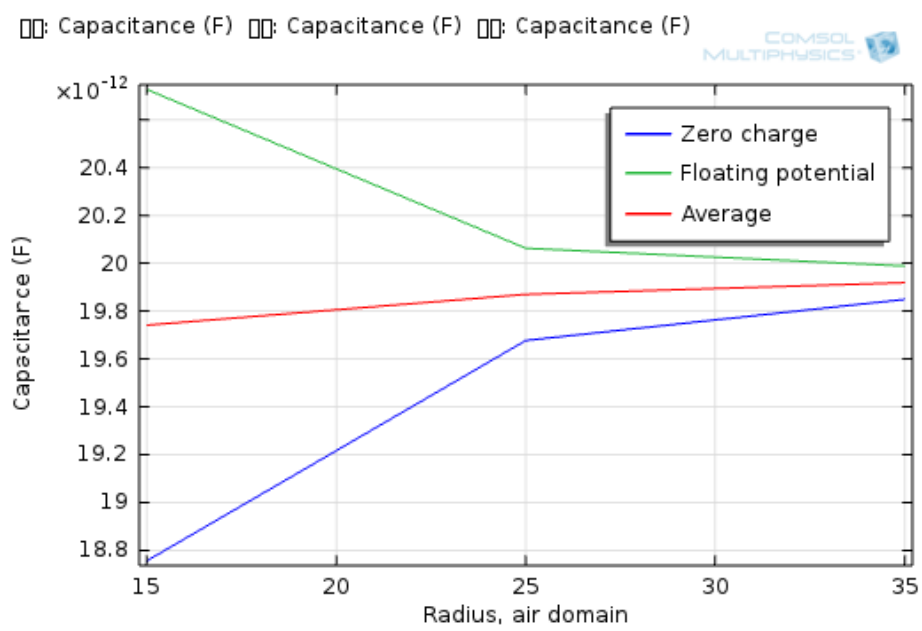

\section{CONCLUSIONS}

Figure 4: Convergence of the capacitance as the size of the surrounding air sphere

This paper firstly introduces the theoretical basis of capacitance sensor. In order to accurately predict the capacitance, this paper then introduces a capacitive sensor model with different air domains, which is influenced by the size of air domain by Comsol software. Simulation results are discussed under different air domains, whose results show that the edge field used in the domain model must be large enough and appropriate boundary conditions must be used. Meanwhile, it can be more convenient to directly familiar with the characteristics of capacitance sensor and to provide a reference for the follow-up testing system design.

\section{ACKNOWLEDGEMENT}

This paper was supported by the Scientific Research Project of Hubei Province (NO. B2015302).

\section{REFERENCES}

[1] Yandong, L., Enxue, Z., Deshuai, D. 2015. Simulation of capacitance sensor based on COMSOL. Journal of electrical and electronic teaching, 37(1), 74-78.

[2] Olmos, A.M., Primicia, J.A., Marron, J.L.F. 2007. Simulation design of electrical capacitance tomography sensors. IET Science, Measurement and Technology, 1(4), 216-223.

[3] Chen, D., Wang, L., Chen, Y. 2007. Analysis and Simulation of Sensor Structure Parameters and Filling Materials Characteristics for Electrical Capacitance Tomography System. Proceedings of The Fifth International Conference on Physical and Numerical Simulation of Materials Processing. [4] Feng, J., Xiaohua, W., Yuping, Z., Qingchang, L. 2003. Simulation of the Capacitance Sensor about its Electric Field Distribution, China, Shenyang.

[5] Ii, B., Wang, Y., Yang, G., Yuan, J. 2014. Finite Element Simulation and Optimization Design of Capacitance Sensor with Loop Test Plate. China, Changsha.

[6] Dong, Z., Sheng-liang, C. 2008. Dynamic characteristic analysis of capacitance sensor based on Matlab. Journal of Chongqing University of Arts and Sciences.

[7] Ke, D., Hong, Y., Xiao-yun, W. 2015. Research on the Simulation of Geometric Optics Based on COMSOL Multiphysics Software. Science and Technology of West China, 4 (10), 95-97. 
[8] XiaoHui, H., Xu Z., Ming, L., YuanFang, C., Peng, L., WeiHua, P., Chun, Z., HongDa, C., 2014. A flexible capacitive tactile sensor array with micro structure for robotic application. Science China (Information Sciences), 57 (12), 1-6.

[9] Fan, L., Hao, W., DengHua, Y., DongDong, Z., WeiHua, X. 2013. Application of profile likelihood function to the uncertainty analysis of hydro meteorological extreme inference. Science China (Technological Sciences), 56 (12), 3151-3160.

[10] Moreno, G.A., Lake, L.W. 2014. On the uncertainty of interwall connectivity estimations from the capacitance-resistance model. Petroleum Science, 2, 265-271. 